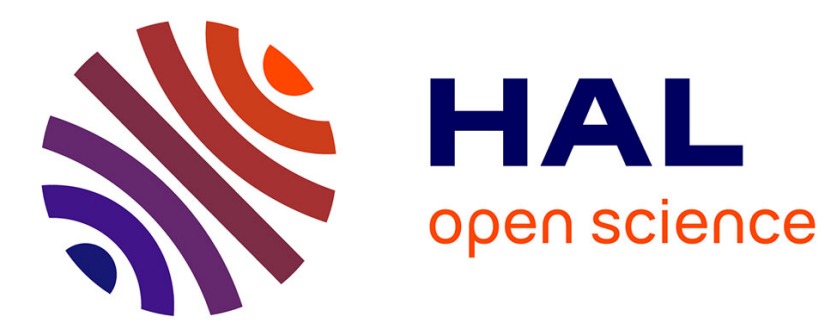

\title{
Polarity determination for GaN thin films by electron energy-loss spectroscopy
}

\author{
X. Kong, G. Q. Hu, X. F. Duan, Y. Lu, X. L. Liu
}

\section{To cite this version:}

X. Kong, G. Q. Hu, X. F. Duan, Y. Lu, X. L. Liu. Polarity determination for GaN thin films by electron energy-loss spectroscopy. Applied Physics Letters, 2002, 81 (11), pp.1990-1992. 10.1063/1.1504876 . hal-01759265

\section{HAL Id: hal-01759265 \\ https://hal.science/hal-01759265}

Submitted on 5 Apr 2018

HAL is a multi-disciplinary open access archive for the deposit and dissemination of scientific research documents, whether they are published or not. The documents may come from teaching and research institutions in France or abroad, or from public or private research centers.
L'archive ouverte pluridisciplinaire HAL, est destinée au dépôt et à la diffusion de documents scientifiques de niveau recherche, publiés ou non, émanant des établissements d'enseignement et de recherche français ou étrangers, des laboratoires publics ou privés. 


\title{
Polarity determination for GaN thin films by electron energy-loss spectroscopy
}

\author{
X. Kong, ${ }^{\text {a) }}$ G. Q. Hu, and X. F. Duan \\ Beijing Laboratory of Electron Microscopy, Institute of Physics \& Center for Condensed Matter Physics, \\ Chinese Academy of Sciences, P.O. Box 2724, Beijing 100080, People's Republic of China \\ Y. Lu and X. L. Liu \\ Laboratory of Semiconductor Materials Science, Institute of Semiconductors, Chinese Academy of Sciences, \\ P.O. Box 912, Beijing 100083, People's Republic of China
}

(Received 6 May 2002; accepted for publication 10 July 2002)

\begin{abstract}
The intensity of the $\mathrm{N} K$ edge in electron energy-loss spectra from a $\mathrm{GaN}$ thin film shows a pronounced difference when the orientation of the film approaches the (0002) and (000-2) Bragg reflections, along the polar direction. This experimental result can be interpreted by the effect associated with interference between the Bloch waves of the incident electron in the GaN crystal. The theoretical calculations indicate that, at the Bragg condition of $g=0002$ along the $\mathrm{Ga}-\mathrm{N}$ bond direction, the thickness-averaged electron current density on the $\mathrm{N}$ atom plane is much higher than that at $g=000 \overline{2}$, with a maximum as the specimen thickness is about $0.4 \xi_{0002}$ (the two-beam extinction distance). The delocalization effect on the experimental spectra is also discussed.
\end{abstract}

(C) 2002 American Institute of Physics. [DOI: 10.1063/1.1504876]

In III-V semiconductors, the polarity in a noncentrosymmetric structure is related to the stacking sequence of the (0001) atomic planes in wurtzite-type GaN, or the (111) planes in zinc blende-type GaAs, for example. It is of major importance for surface morphology, surface stability, and electronic properties of these semiconductors. Determination of the polarity can be carried out conventionally by convergent-beam electron diffraction (CBED) in transmission electron microscopy (TEM). ${ }^{1-4}$ Since the CBED pattern is sensitive to the defects and the thickness in the illuminated area of the specimen, the use of this method is sometimes limited. The atomic location by channeling-enhance microanalysis technique is an alternative method to determine the polarity, ${ }^{5}$ based on the interference between the Bloch waves in the crystal. ${ }^{6}$ Recently, Jiang et al. demonstrated the method using x-ray energy dispersion spectroscopy (EDS). ${ }^{7}$ The polarity can be determined by comparison of two or more element-characteristic x-ray intensities, which will always enhance at one Bragg orientation, and diminish at the opposite. However, the count rate of characteristic $\mathrm{x}$ rays is very low because the fluorescence yield $\omega$ is very small. The fraction recorded by an EDS detector is about $1 \%$ for a very small collection solid angle. In contrast, the inelastically scattered electrons with an energy loss of $E$ are concentrated within a cone of semiangle $\theta_{E},\left(\theta_{E}=E / 2 \gamma E_{0}\right.$ is the characteristic angle, $\gamma$ is the relativistic factor, and $E_{0}$ is the original energy of the incident electrons), and a large fraction of the electrons that contain information about an inner-shell ionization can be collected and analyzed by electron energyloss spectroscopy (EELS). Taft $\varnothing$ discussed the reciprocity in electron energy-loss (EEL) spectra from GaAs, and observed a much larger difference in EELS than in EDS for pairs of experimental arrangements related by a mirror operation

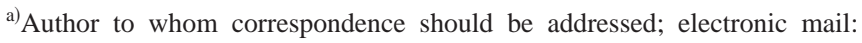
xkong@blem.ac.cn
}

around the polar (111) planes. ${ }^{8}$ EELS provides a more sensitive way to investigate the polarity of a noncentrosymmetric crystal.

In this letter, we use the EELS to determine the polarity of a $\mathrm{GaN}$ thin film deposited on a (111) $\mathrm{Si}$ substrate. The experimental result is in good agreement with the theoretical calculation based on the electron dynamic diffraction at the two-beam condition. The delocalization effect is also discussed, as it is a sensitive factor in the experiment to determine the polarity, especially for the low-loss electrons. ${ }^{9-12}$

The GaN thin film was deposited on a (111) silicon substrate with a (0001) AlN buffer layer, using metalorganic chemical vapor deposition. The direction of the cation $(\mathrm{Ga})$ to the anion $(\mathrm{N})$ is defined as [0001] in the real space. ${ }^{1-4,7}$ The EELS experimental studies were carried out using a Philips CM200 field emission gun TEM equipped with a Gatan image filtering system, operated at $200 \mathrm{kV}$. The energy resolution is about $1.0 \mathrm{eV}$ according to the measured full width at half maximum of the zero-loss peak. In the diffraction mode, the cross-sectional sample was titled to the Bragg position of either $(0002)$ or $(000 \overline{2})$, and away from any zone axis. The EEL spectra were collected with an off-axis collection aperture of about $3 \mathrm{mrad}$ collection semiangle, and positioned at the middle of the incident beam and the diffraction beam. The thickness of the area chosen for observation was estimated to be $18-20 \mathrm{~nm}$, as obtained from low-loss spectrum analysis. ${ }^{13}$ It is less than half of the two-beam extinction distance $\xi_{0002}$, which is about $56 \mathrm{~nm}$. In order to minimize the instability in the high tension supply of the microscope and to decrease the statistic error, the spectrum including $\mathrm{N} K$-edge and $\mathrm{Ga} L$ edges was acquired by accumulating 20 times using a $0.5 \mathrm{~s}$ acquisition time. Figure 1 shows the spectra with the background of $\mathrm{N} K$-edge subtracted, obtained at (0002) and $(000 \overline{2})$ Bragg orientations. It is obvious that the $\mathrm{N} K$ peak has a relatively higher intensity at the Bragg orientation of (0002) than that at the Bragg 


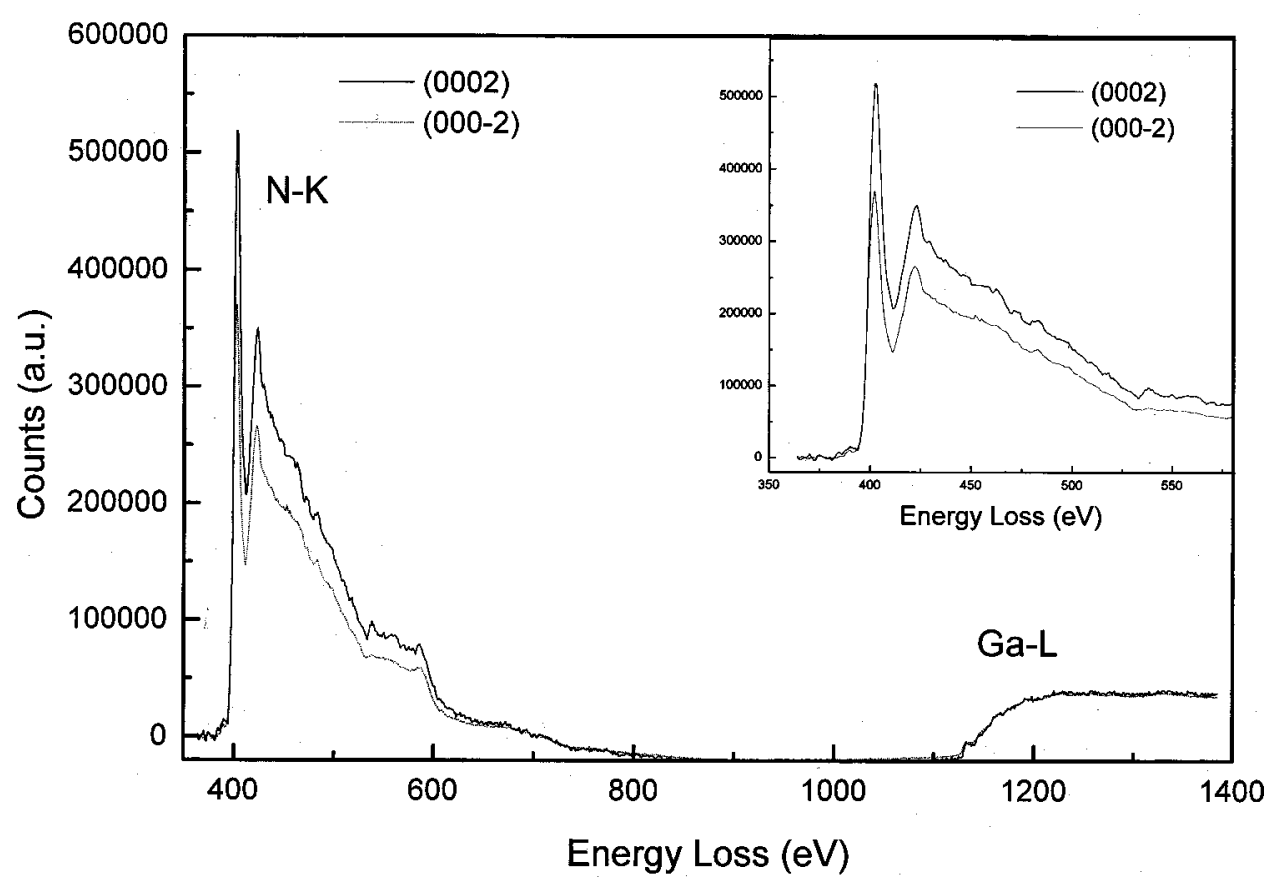

FIG. 1. Comparison of the EEL spectra acquired at (0002) and $(000 \overline{2})$ Bragg conditions. The inset is the magnified N $K$ edge.

orientation of $(000 \overline{2})$. Three other pairs of spectra were recorded from the areas with thicknesses of about $0.57,1.23$, and $1.84 \xi_{0002}$, respectively. All the experimental results show that the $\mathrm{N}$ to $\mathrm{Ga}$ ratio is higher at the Bragg position of (0002) than that at the Bragg position of $(000 \overline{2})$ from the same area.

Inside the thin crystal, the fast electrons propagate as a superposition of Bloch states. ${ }^{14}$ At the two-beam condition, the thickness-averaged electron distribution is localized near some special atomic planes, as a consequence of the interference between the Bloch waves. The thickness-averaged electron current intensity (TAECD) close to Bragg condition $\bar{g}$, without absorption, has been given as ${ }^{7,15}$

$$
\begin{aligned}
I(r)= & \frac{1}{t} \int_{0}^{t}|\psi(z)|^{2} d z \\
= & 1-\frac{1}{\sqrt{1+w^{2}}} \cdot \frac{1-\cos \left(2 \pi t^{\prime} \sqrt{1+w^{2}}\right)}{2 \pi t^{\prime} \sqrt{1+w^{2}}} \\
& \times \sin \left(2 \pi \vec{g} \cdot \vec{r}+\varphi_{g}\right) \\
& -\frac{w}{1+w^{2}}\left(1-\frac{\sin \left(2 \pi t^{\prime} \sqrt{1+w^{2}}\right)}{2 \pi t^{\prime} \sqrt{1+w^{2}}}\right) \\
& \times \cos \left(2 \pi \vec{g} \cdot \vec{r}+\varphi_{g}\right),
\end{aligned}
$$

where $\psi(\mathrm{z})$ is the electron wave function in the crystal, the orientation of the specimen is represented by $w\left(w=s_{g} \xi_{g}\right.$, the deviation parameter, and the two-beam extinction distance, respectively), the thickness is expressed in units of the two-beam extinction distance $\left(t^{\prime}=t / \xi_{g}\right), \vec{r}$ is the atomic position in a unit cell, and $\varphi_{g}$ is the phase shift due to the absence of a center of symmetry. Figures 2(a) and 2(b) show the profiles of TAECD versus the atomic planes position at (0002) and $(000 \overline{2})$ Bragg conditions, respectively, for a Downloaded 06 Aug 2005 to 129.20.82.156. Redistribution subject sample thickness of $0.4 \xi_{g}$. The maximum of the intensity curve for (0002) Bragg condition is around the position of the $\mathrm{N}$ atomic plane, and while at the $(000 \overline{2})$ Bragg condition the intensity reaches a minimum. Around the Ga atomicplane position, the electron intensities are roughly equal. This indicates that the $\mathrm{N} K$ edge taken at the (0002) Bragg condition should be higher than that at the $(000 \overline{2})$ Bragg condition when the spectra are normalized using the Ga $L$ edges. The experimental result is in good agreement with the
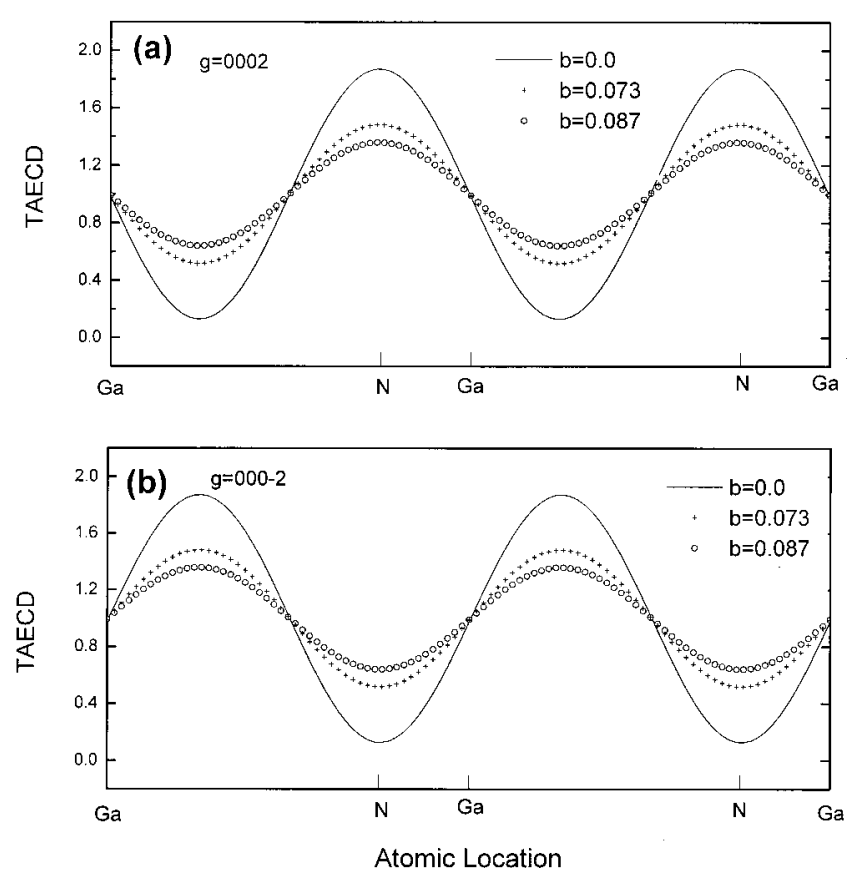

FIG. 2. The calculated thickness-averaged electron current density across a unit cell at (0002) (a) and $(000 \overline{2})$ (b) Bragg conditions for the impact parameter $b=0.0 \mathrm{~nm}$ (without delocalization), $0.073 \mathrm{~nm}$, and $0.087 \mathrm{~nm}$ using Eq. (4). The sample thickness is $0.4 \xi_{0002}$. In (a) the maximum and in (b) the minimum is near the $\mathrm{N}$ atomic planes and they will give the largest TAECD difference.

to AIP license or copyright, see http://apl.aip.org/apl/copyright.jsp 
theoretical calculation shown in Fig. 1. Using this criterion, the polarity of the GaN thin film can be determined directly using EELS.

Similar to the case in the x-ray EDS experiment, several experimental factors should be considered in the EELS experiment, such as the orientation of the sample deviated from the exact two-beam Bragg condition, beam convergence effects, and the effect of absorption. ${ }^{7,16}$ Furthermore, the delocalization of inelastic scattering associated with the width of the interaction potential is an important factor, which may affect the determination of the polarity, especially when using a low energy-loss characteristic edge. The delocalization is represented by the impact parameter $b$ of the incident electron. The scatter event is more localized if the collection aperture is positioned off axis, ${ }^{13}$ as in our experiment. Usually, root-mean-square impact parameter $b_{\mathrm{RMS}}$ can be used to estimate the delocalization. Pennycook proposed that $b_{\mathrm{RMS}}$ could be given as ${ }^{9}$

$$
b_{\mathrm{RMS}}=\frac{h \nu}{E_{i}}\left(\ln \frac{4 E_{0}}{E_{i}}\right)^{-1 / 2},
$$

where $\nu$ is the velocity of the incident electron and $E_{i}$ is the binding energy of the electron in the inner shell. Another expression of $b_{\mathrm{RMS}}$, as proposed by Bourdillon, is

$$
b_{\mathrm{RMS}}=\frac{f \lambda}{2 \pi \theta_{E}},
$$

where $f=0.5^{13,17,18}$ and $\lambda$ is the wavelength of the incident electron. In our experiment, the root-mean-square impact parameter for the $\mathrm{N} K$ edge was estimated to be about 0.073 and $0.087 \mathrm{~nm}$ by Eqs. (2) and (3), respectively. In order to consider this delocalization effect on the TAECD, formula (1) is modified as

$$
\begin{aligned}
I^{b}(r)= & \frac{1}{2 b} \int_{r-b}^{r+b} I(r) d r \\
= & 1-\left\{\frac{1}{\sqrt{1+w^{2}}} \sin (2 \pi \vec{g} \cdot \vec{r}\right. \\
& \left.+\varphi_{g}\right) \frac{1-\cos \left(2 \pi t^{\prime} \sqrt{1+w^{2}}\right)}{2 \pi t^{\prime} \sqrt{1+w^{2}}}-\frac{w}{1+w^{2}} \cos (2 \pi \vec{g} \cdot \vec{r} \\
& \left.\left.+\varphi_{g}\right)\left(1-\frac{\sin \left(2 \pi t^{\prime} \sqrt{1+w^{2}}\right)}{2 \pi t^{\prime} \sqrt{1+w^{2}}}\right)\right\} \\
& \times \frac{\sin (2 \pi g b)}{2 \pi g b},
\end{aligned}
$$

with $b=b_{\text {RMS }}$. Figures 2(a) and 2(b) show the TAECD on N atomic planes at $(0002)$ and $(000 \overline{2})$ Bragg conditions, for different impact parameters. Obviously, the delocalization effect will dampen the interference of the Bloch states.

In order to estimate the thickness at which the change in the intensity of the $\mathrm{N} K$ edge is maximum, we calculated the difference of the pair of TAECD at (0002) and (000) Bragg conditions, shown in Fig. 3. The calculations indicate that the

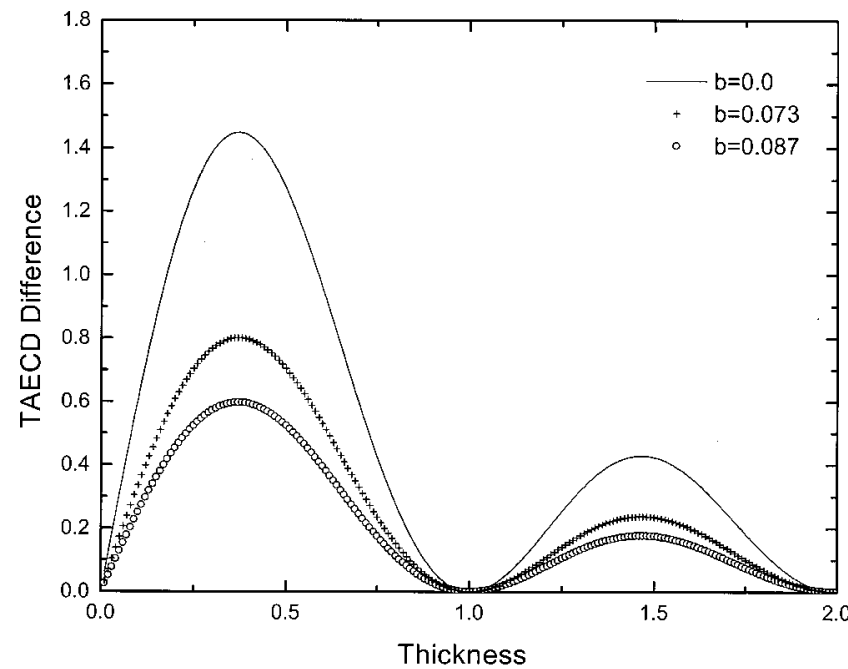

FIG. 3. The difference of the calculated averaged-thickness electron current density vs the sample thickness. The thickness is expressed in units of the two-beam extinction distance $\xi_{0002}$. The difference of the calculated averaged-thickness electron current density does not change sign when the thickness changes.

difference has a maximum at a thickness of $0.4 \xi_{g}$, and turns to be zero at thicknesses of $n \xi_{0002}$ (when $n$ is an integer). It means that the best result would be obtained from an area with thickness in the range of $0.3-0.5 \xi_{0002}$, about $25 \mathrm{~nm}$. The oscillations are dampened as the thickness increased. The calculations show that the TAECD on the $\mathrm{N}$ atomic plane is always larger at the (0002) Bragg condition than that at the $(000 \overline{2})$ Bragg condition.

This work was supported by the National Natural Science Foundation of China Grant No. 50072044.

${ }^{1}$ F. A. Ponce, D. P. Bour, W. Y. Young, M. Saunders, and J. W. Steeds, Appl. Phys. Lett. 69, 337 (1996).

${ }^{2}$ B. Daudin, J. L. Rouviere, and M. Arlery, Appl. Phys. Lett. 69, 2480 (1996).

${ }^{3}$ J. L. Rouviere, J. L. Weyher, M. Seelmann-Eggebert, and S. Porowski, Appl. Phys. Lett. 73, 668 (1998).

${ }^{4}$ P. D. Han, Zh. G. Wang, X. F. Duan, and Z. Zhang, Appl. Phys. Lett. 78, 3974 (2001).

${ }^{5}$ J. C. H. Spence and J. Taftø, J. Microsc. 130, 147 (1983).

${ }^{6}$ J. Taftø, Phys. Rev. Lett. 51, 654 (1983).

${ }^{7}$ N. Jiang, T. J. Eustis, J. Cai, F. A. Pronce, J. C. H. Spence, and J. Silcox, Appl. Phys. Lett. 80, 389 (2002).

${ }^{8}$ J. Taft $\varnothing$, Acta Crystallogr., Sect. A: Found. Crystallogr. A43, 208 (1987).

${ }^{9}$ S. J. Pennycook, Ultramicroscopy 26, 239 (1988).

${ }^{10}$ K. M. Krishnan, P. Rez, and G. Thoms, Acta Crystallogr., Sect. B: Struct. Sci. 41, 396 (1985).

${ }^{11}$ Z. Horita, H. Kuninaka, T. Sano, M. Nemoto, and J. C. H. Spence, Philos. Mag. A 67, 425 (1993).

${ }^{12}$ D. A. Muller and J. Silcox, Ultramicroscopy 59, 195 (1995).

${ }^{13}$ R. F. Egerton, Electron Energy Loss Spectroscopy in the Electron Microscope, 2nd ed. (Plenum, New York, 1996).

${ }^{14}$ P. Hirsch, A. Howie, R. B. Nicholson, D. W. Pashley, and M. J. Whelan, Electron Microscopy of Thin Crystal, 2nd ed. (Huntington, New York, 1977)

${ }^{15}$ J. C. H. Spence and J. M. Zuo, Electron Microdiffraction (Plenum, New York, 1992).

${ }^{16}$ L. J. Allen, Ultramicroscopy 48, 97 (1993).

${ }^{17}$ M. J. Seaton, Proc. Phys. Soc. London 79, 1105 (1962).

${ }^{18}$ A. J. Bourdillon, P. G. Self, and W. M. Stobbs, Philos. Mag. A 44, 1335 (1981). 